\title{
Apatinib induced ferroptosis by lipid peroxidation in gastric cancer
}

\author{
Liying Zhao ${ }^{1}$-Yanmei Peng ${ }^{1} \cdot$ Sixiao $\mathrm{He}^{2} \cdot \mathrm{Ru} \mathrm{Li}^{2} \cdot$ Ziqing Wang $^{2} \cdot$ Junhao Huang $^{2} \cdot$ Xuetao Lei $^{1} \cdot$ Guoxin $\mathrm{Li}^{1}$. \\ Qiang $\mathrm{Ma}^{2}$
}

Received: 26 May 2020 / Accepted: 28 December 2020 / Published online: 5 February 2021

(c) The International Gastric Cancer Association and The Japanese Gastric Cancer Association 2021

\begin{abstract}
Background Apatinib, a competitive inhibitor of VEGFR2, has anti-angiogenesis and anticancer activities through different mechanisms, but it still cannot fully explain the drug efficacy of apatinib. Ferroptosis, associated with lethal lipid peroxidation, has emerged to play an important role in cancer biology, however, the exact role of ferroptosis in apatinib-mediating anticancer treatment are still not clear.

Methods The effects of (1S, 3R)-RSL3 and apatinib were evaluated in different GC cell lines and in normal human gastric epithelial cells. Further, the effects of apatinib and inhibition of antioxidant defense enzyme glutathione peroxidase (GPX4) on cell viability, cell death, glutathione (GSH) levels, lipid ROS production, cellular malondialdehyde (MDA) levels and protein expression were evaluated in vitro as well as in a mouse tumor xenograft model. The expression level of GPX4 in GC tissues and paracancerous tissues was measured by immunohistochemistry.

Results (1S, 3R)-RSL3 selectively killed GC cells, but not normal cells. Apatinib induced ferroptosis in GC cells by decreasing cellular GSH levels and increasing lipid peroxidation levels. This effect was blocked by co-incubation with ferrostatin-1, liproxstatin-1, GSH, or vitamin E. Further investigation revealed that apatinib down-regulated GPX4 expression via inhibition of the transcription factors Sterol regulatory element-binding protein-1a (SREBP-1a). Besides, we found that multi-drug resistant GC cells were vulnerable to apatinib-induced GPX4 inhibition.

Conclusions In summary, we show that apatinib could induce the lipid peroxidation through GPX4 mediated by SREBP-1a, then negatively regulate the GC cell, even the multi-drug-resistant GC cell, ferroptosis.
\end{abstract}

Keywords Gastric cancer · Apatinib · Ferroptosis · GPX4 · Lipid peroxidation

$\begin{array}{ll}\text { Abbreviations } \\ \text { GC } & \text { Gastric cancer } \\ \text { GPX4 } & \text { Glutathione peroxidase } 4 \\ \text { GSH } & \text { Glutathione } \\ \text { GSSG } & \text { Glutathione disulfide } \\ \text { ROS } & \text { Reactive oxygen species } \\ \text { RSL3 } & \text { RAS-selective lethal3 } \\ \text { MDA } & \text { Malondialdehyde } \\ \text { Fer-1 } & \text { Ferrostatin-1 }\end{array}$

Liying Zhao and Yanmei Peng contributed equally to this article.

Guoxin Li

gzliguoxin@163.com

1 Department of General Surgery, Nanfang Hospital, The First School of Clinical Medicine, Southern Medical University, Guangzhou 510515, China

2 Department of Biopharmaceutics, School of Laboratory Medicine and Biotechnology, Southern Medical University, Guangzhou 510515, China

\author{
DFO Deferoxamine \\ Lip-1 Liproxstatin-1 \\ siRNA Short interfering RNA \\ PBS Phosphate-buffered saline
}

\section{Background}

Apatinib is a highly selective inhibitor of vascular endothelial growth factor receptor-2 (VEGFR2) tyrosine kinase, and was the first anti-angiogenic agent approved for treatment of metastatic GC by cFDA [1]. Apatinib has been shown to promote survival rates as a second-line treatment for patients with advanced or metastatic GC who progressed during treatment with fluoropyrimidine- or platinum-based firstline chemotherapies [2]. Studies confirmed that apatinib, has anti-angiogenesis and anticancer activities through different mechanisms such as inducing apoptosis and inhibiting proliferation and metastasis [3]. Apatinib can be used as an 
adjuvant for cancer treatment through acting on $\mathrm{ABC} 1$ and $\mathrm{ABC} 2$ pumps, in addition, it can be also used to inhibit the formation of vascular mimic structures that makes this drug preferable from the others, however, it still cannot predict the drug efficacy of apatinib $[4,5]$. Therefore, the mechanism of apatinib anticancer treatment needs to be explored.

Cytoplasmic membrane lipid peroxidation, an autooxidative process initiated by the attack of free radicals, contributes to the progression of various types of regulated cell death [6]. Ferroptosis, a recently discovered form of regulated, programmed cell death associated with lethal lipid peroxidation, is distinct from apoptosis, necroptosis, and autophagic cell death [7]. Ferroptosis may be a promising tumor suppression mechanism with therapeutic potential against cancer [8,9]. However, it remains unknown whether ferroptosis induced by lipid peroxidation contributes to apatinib-mediated anticancer treatment. Here we demonstrated that that glutathione peroxidase 4 (GPX4), an antioxidant defense enzyme active in repairing oxidative damage to lipids, is an important negative regulator of GC cells ferroptosis. Genetic inactivation of GPX4 increases lipid peroxidation, thus exacerbating apatinib-mediated ferroptosis in GC cells as well as tumor xenografts in mice. Thus, our findings uncover a critical mechanism that controls the lipid peroxidation in the context of apatinib-mediated anticancer treatment.

\section{Methods}

\section{Gastric cancer cell lines and cell culture}

The GC cell lines MGC803, MKN45, BGC823, SGC7901, and AGS were obtained from American Type Culture Collection (ATCC, Manassas, VA, USA). Cisplatin-resistant and 5-FU-resistant MGC-803/DDP/5-FU were developed from MGC-803 cells. All cells were cultured in Dulbecco's Modified Eagle's Medium (DMEM) supplemented with 10\% FBS (Gibco) in a $37{ }^{\circ} \mathrm{C}$ humidified incubator with a $5 \% \mathrm{CO}_{2}$ atmosphere. To maintain the MDR phenotype, MGC-803/ DDP/5-FU cells were cultured with $5 \mu \mathrm{g} / \mathrm{ml} 5$-FU and $5 \mu \mathrm{g} /$ $\mathrm{ml}$ cisplatin (DDP).

\section{Cell viability assay}

Cell viability following exposure to combinations of RSL3 (1-15 $\mu \mathrm{M}$, Sigma-Aldrich), apatinib (20-40 $\mu \mathrm{M}$, Hengrui), ferrostatin-1 (10 $\mu \mathrm{M}$, Sigma-Aldrich), and or liproxstatin-1 (1-10 $\mu \mathrm{M}$, Sigma-Aldrich SML1414), with or without suppression of GPX4 expression, was assessed using the CCK8 kit.

\section{Transmission electron microscopy}

After $24 \mathrm{~h}$ of treatment with $20 \mu \mathrm{M}$ apatinib, GC cells were digested using $0.25 \%$ trypsin, and then fixed with $1.5 \%$ glutaraldehyde. Ultrathin sections ( $80 \mathrm{~nm}$ ) were cut and imaged using a Tecnai TF20 TEM with an AMT XR41 camera. Transmission electron microscopy images were acquired using an AFEI Tecnai 20200 kV FEG Electron Microscope.

\section{Lipid peroxidation analysis using imaging and flow cytometry}

For imaging, GC cells were seeded at 200,000 cells per well in six-well dishes, then incubated with $20 \mu \mathrm{M}$ apatinib or $10 \mu \mathrm{M}$ RSL3 for $24 \mathrm{~h}$. During the last hour of incubation, $1 \mu \mathrm{g} / \mathrm{mL}$ Hoechst 33,342 and $1 \mu \mathrm{M}$ BODIPY-581/591 C11 were added to the culture medium for live-cell imaging. For flow cytometry analysis, GC cells were treated with DMSO or apatinib $(20 \mu \mathrm{M})$ for $24 \mathrm{~h}$. For the final $30 \mathrm{~min}$, the cells were incubated with $5 \mu \mathrm{M}$ BODIPY-C11 or $10 \mu \mathrm{M}$ DCF-DA.

\section{Tissue microarray}

A constructed tissue microarray (TMA) containing 89 gastric cancer cases was obtained from Servicebio (Wuhan, China). Postsurgical pathological staging and overall survival time results were obtained for all cases (Supplementary Table 1). These parameters were used to evaluate clinical relevance of the results.

\section{Quantitation of mRNA by real-time PCR}

Total RNA was extracted from cells and purified using TRIzol Reagent (Takara) according to the manufacturer's instructions. Complementary DNA was synthesized using the PrimeScriptTM RT reagent kit (Takara). The primers used in this study are listed in Supplementary Table 2.

\section{Immunohistochemistry (IHC)}

Sections were incubated with anti-GPX4 (1:100 dilution). The sections were scored for staining intensity according to the following scale: 0 (no staining), 1 (weak staining, light yellow), 2 (moderate staining, yellowish brown), and 3 (strong staining, brown), with 0 and 1 considered low expression, and 2 and 3 considered high expression. 


\section{Gene knockdown using siRNA}

Small interfering (si) GPX4 and control siRNAs were obtained from GenePharma Biotech (Suzhou, Jiangsu, China). The sequences are listed in Supplementary Table 3. Gastric cancer cells were transfected with $50 \mathrm{nM}$ si-GPX4 or scrambled negative control (si-NC) siRNA using siRNA mate (GenePharma) and incubated for $48 \mathrm{~h}$ for further experiments.

\section{ChIP assay}

ChIP was carried out according to the manufacturer's protocol specified with the ChIP Kit (ab500; Abcam). Quantitation of immunoprecipitated genomic DNA regions was performed with real-time PCR, The primers are listed in Supplementary Table 2.

\section{Promoter luciferase assay}

Gastric cancer cell lines were co-transfected using Lipofectamine 2000 kit (Life Technologies) as per the manufacturer's instructions, with GPX4 promoter-reporter sequences and a pEZX-FR01 plasmid (GeneCopoeia) to control for transfection efficiency.

\section{Tumor xenografts}

Female nude mice (BALB/c, nu/nu, 18-22 g, 4-5 weeks old) were obtained from Guangdong Medical Laboratory Animal center, China, and maintained under specific pathogen-free conditions on a $12 \mathrm{~h} / 12 \mathrm{~h}$ light/dark cycle. Each mouse was injected subcutaneously with eight million luciferase-expressing cells resuspended in $50 \mu \mathrm{l}$ of PBS and $50 \mu \mathrm{l}$ of Matrigel (BD Biosciences). When a palpable mass had developed, the mice were randomly divided into five groups: apatinib (50 mg/kg/day oral dose for 14 days); RSL3 (100 mg/kg injection of RSL3 twice per week for 2 weeks at the same site); both; apatinib (50 mg/kg/day oral dose for 14 days) plus vitamin E $(100 \mathrm{mg} / \mathrm{kg} / \mathrm{day}$ oral dose for 14 days); and vehicle (DMSO, $100 \mu \mathrm{l}$ oral dose for 14 days). Tumors were quantified using bioluminescence imaging every 4 days, and tumor volumes were measured by determining the lengths $(L)$ and widths $(W)$ of the tumors using a caliper. Tumor volume was calculated according to the following equation: $V=\left(L^{*} W^{*} W\right) / 2$. The mice were sacrificed on the 14th day after treatment. Tumor samples were prepared for Perls staining and IHC analyses.

\section{Statistical analysis}

Statistical analyses were performed using SPSS 20.0 statistical software package (SPSS Inc, Chicago, Illinois, USA).
Quantitative data are presented as the mean \pm SD of three independent experiments, and were analyzed using Pearson's $\chi^{2}$ test or Student's $t$ test. A survival curve was generated using the Kaplan-Meier method, and groups were compared using two-tailed log rank tests and Wilcoxon tests. Cox proportional hazards regression was used to determine the univariate and multivariate hazard ratios for selected potential parameters. $P$ values $<0.05$ (two tailed) were considered to indicate significance.

\section{Results}

\section{Gastric cancer (GC) cell lines were more susceptible to ferroptosis than normal cells}

Yang et al. showed that diffuse large B cell lymphomas and renal cell carcinomas in multiple tissues, such as the large intestine, hematopoietic and lymphoid tissue, lung, ovary, and skin, were susceptible to ferroptosis [10]. We evaluated the sensitivity of GC cells to ferroptosis. Treatment with RSL3 decreased the viability of MGC- 803 cells in a dosedependent manner. Furthermore, cell viability of MGC-803 cells was significantly reduced in response to treatment with $15 \mu \mathrm{M}$ RSL3 compared with that of normal human gastric epithelial (GES-1) cells $(p<0.001)$ (Fig. 1a). RSL3 induced accumulation of lipid radicals in MGC-803 cells, but not in GES-1 cells, as determined using BODIPY-C11 (Fig. 1b). In addition, the viability of RSL3-treated AGS, MGC-803, BGC-823, and SGC-7901cells decreased in a time-dependent manner, and this effect was inhibited by coincubation with ferrostatin-1 $(10 \mu \mathrm{M})(p<0.05)$ (Fig. 1c).

\section{Apatinib induced ferroptotic cell death in GC cell lines}

Apatinib significantly decreased the number of GC cells, and this decrease was blocked by co-incubation with ferrostatin-1 $(10 \mu \mathrm{M})(p<0.05)$ (Fig. 2a). To determine the form of cell death responsible for this decrease in cell number, several compounds known to inhibit different forms of apoptosis, pyroptosis, necrosis, and autophagy were used. We showed that apatinib-induced cell death was suppressed by pretreatment with the iron chelator deferoxamine (DFO) but not with caspase inhibitors (AcYVAD-CMK), RIPK1 (necrostatin-1), or chloroquine (CQ) (Fig. 2b). Iron assay kit results showed significantly higher oncentrations of total and ferrous iron in GC cells treated with $20 \mu \mathrm{M}$ apatinib compared with the DMSO group ( $p<0.01)$ (Fig. 2c). Furthermore, MGC-803 cells treated with $20 \mu \mathrm{M}$ apatinib for $24 \mathrm{~h}$ showed reduced cell viability, and co-incubation with Lip-1 blocked this effect $(p<0.05)$ (Fig. 2d). Ferroptotic cell death was confirmed 
$\mathbf{a}$

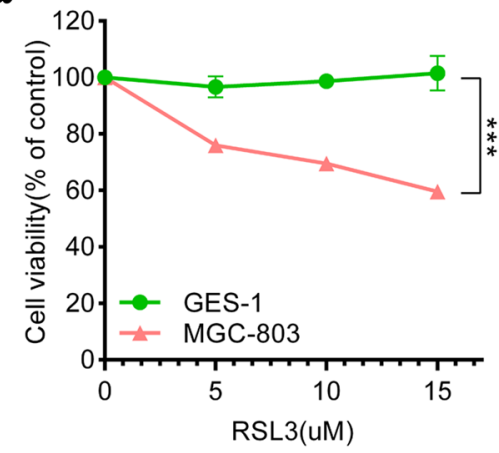

b

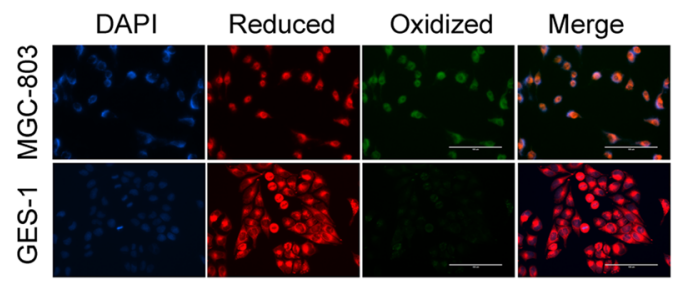

c
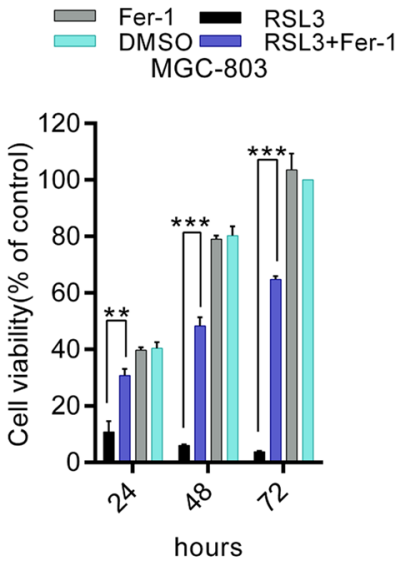

AGS

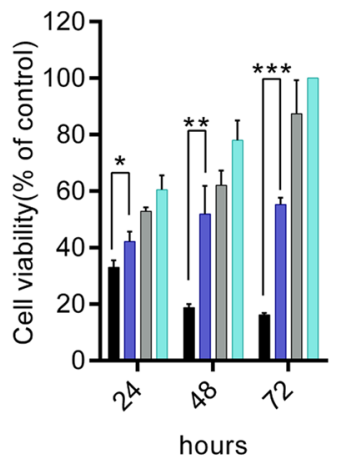

Fig. 1 Gastric cancer (GC) cell lines were susceptible to ferroptosis. a Cell viability was assessed following exposure of MGC-803 cells and normal human gastric epithelial (GES-1) cells to different concentrations of (1S, 3R)-RSL3 $(0-15 \mu \mathrm{M})$ for $12 \mathrm{~h}$. b Immunofluorescence staining of oxidized lipid ROS (green color) and reduced lipid ROS (red color) formation in MGC-803 and GSE-1 cells treated with

using transmission electron microscopy (TEM). Incubation of MGC-803 cells with apatinib for $24 \mathrm{~h}$ resulted in an increase in mitochondrial membrane density and smaller mitochondria compared with those in cells that were not treated with apatinib (Fig. 2e). Apatinib induced accumulation of lipid radicals in MGC-803 cells, but not in GES-1 cells, as determined using BODIPY-C11, which further confirmed ferroptosis-mediated cell death (Fig. 2f). Total ROS and lipid ROS levels were measured by flow cytometry using the fluorescent probes H2DCFDA and C11-BODIPY, respectively. Treatment with $20 \mu \mathrm{M}$ apatinib for $24 \mathrm{~h}$ increased total ROS and lipid ROS in MGC803 cells, and this increase was significantly inhibited by co-incubation with ferrostatin-1 (10 $\mu \mathrm{M})$ (Fig. 2g). Moreover, MDA levels were increased in apatinib-treated AGS, MGC-803, and MKN45 cells, and this effect was inhibited by co-incubation with ferrostatin-1 (10 $\mu \mathrm{M})$ (Fig. 2h). These findings suggest that apatinib induced ferroptotic cell death in GC cells.

\section{Gastric cancer had low GPX4 expression, which was associated with poor prognosis}

The mechanisms by which apatinib induces ferroptotic cell death in GC cells have not been characterized. Glutathione peroxidase is as a central regulator of ferroptosis. Immunohistochemistry staining was performed using a tissue microarray (TMA) to determine the clinical relevance of GPX4 in GC. Glutathione peroxidase 4 expression was clearly lower in GC tissues than in paracancerous tissues (Fig. 3a, b). Staining was scored on the following scale: negative, 0; weak, 1; moderate, 2; intense, 3 (Fig. 3c). GPX4 expression was identified to be independent prognostic covariates for poor overall survival (Table 1) and Kaplan-Meier survival curves indicated that low expression of GPX4 was associated with poor survival (Fig. 3d). Furthermore, the expression of GPX4 was also associated with TMN stage, as shown in Table 2. 
$\mathbf{a}$

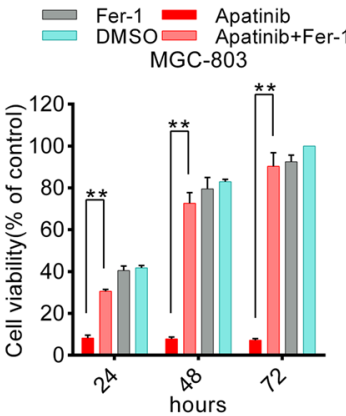

b

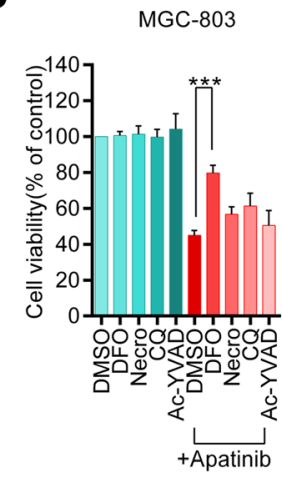

e

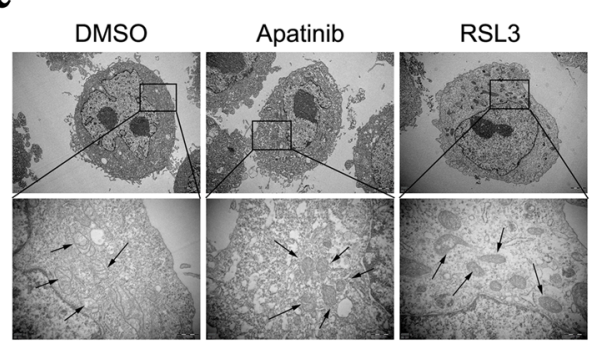

g

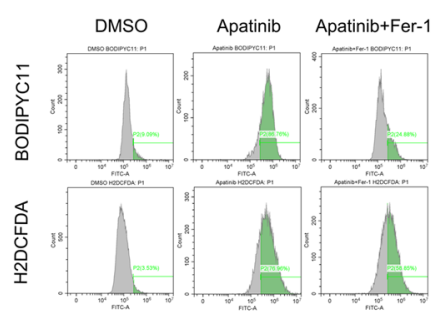

BGC-823

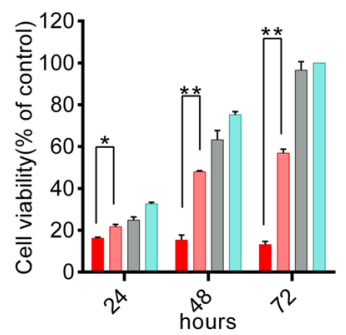

c

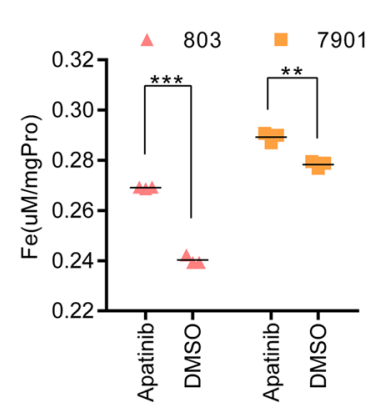

SGC-7901

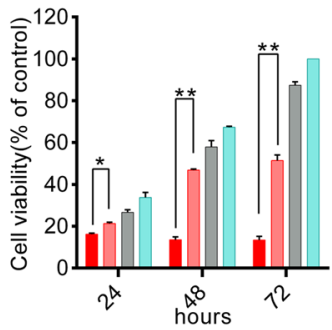

d

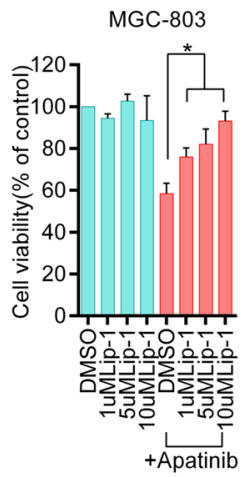

f

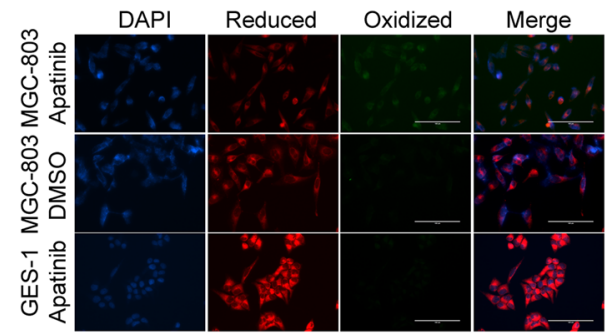

h

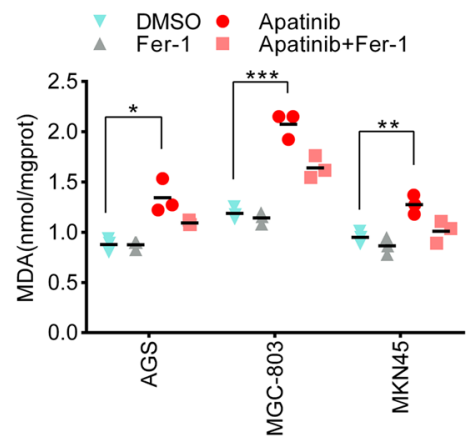

alize MGC-803 cells treated with DMSO, RSL3 $(10 \mu \mathrm{M})$, or apatinib $(20 \mu \mathrm{M})$. Increased mitochondrial membrane density (black arrows) was observed in apatinib- and RSL3-treated MGC-803 cells, but not in the control group. f Immunofluorescence staining of oxidized lipid ROS (green color) and reduced lipid ROS (red color) formation in MGC-803 and GES-1 cells treated with apatinib $(20 \mu \mathrm{M})$, or DMSO for $24 \mathrm{~h}$. Scale bars: $100 \mu \mathrm{m}$. $\mathrm{g}$ Effects of inhibitors on BODIPY C11 and H2DCFDA-sensitive ROS production in MGC-803 cells treated with $20 \mu \mathrm{M}$ apatinib, $10 \mu \mathrm{M}$ Fer-1, or both, for $24 \mathrm{~h}$. h Changes in cellular malondialdehyde levels in GC cell lines treated with $20 \mu \mathrm{M}$ apatinib, $10 \mu \mathrm{M}$ Fer-1, or both. The error bars represent the standard error from three replicates. $* P<0.05$, $* * P<0.01$, $* * * P<0.001$ 
Fig. 3 Glutathione peroxidase 4 expression was down-regulated in GC tissue, and this was associated with poor prognosis. a, c Immunohistochemistry staining of GPX4 in GC samples and non-tumorous tissues $(100 \times$ and $400 \times$ ). b Immunohistochemistry scores of GC samples and non-tumorous tissues. $\mathbf{d}$ Kaplan-Meier curves showed the association of GPX4 expression with overall survival in 89 patients with GC $\mathbf{a}$

c

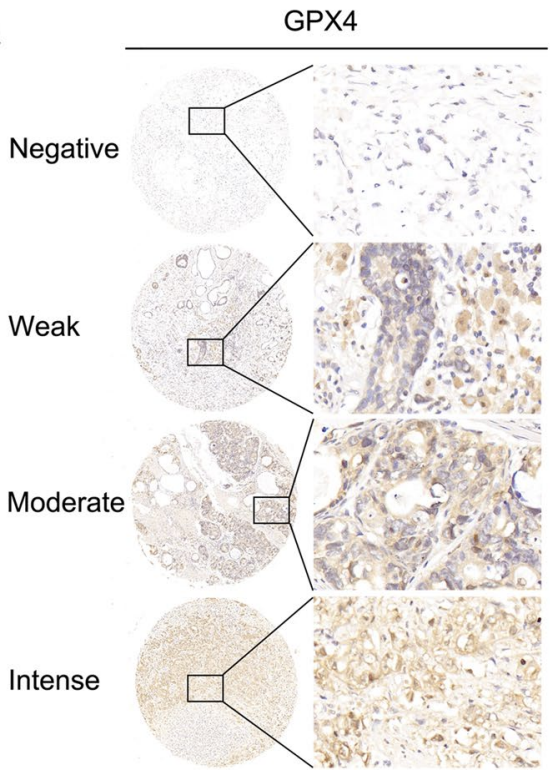

Table 1 Univariate and multivariate analyses of different prognostic factors in 89 patients with GC using Cox regression model

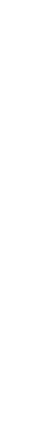

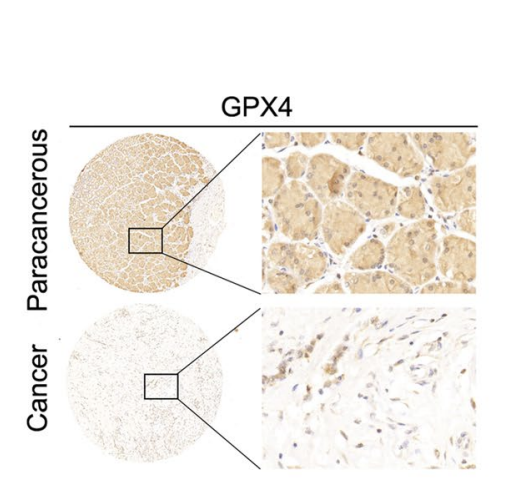

b

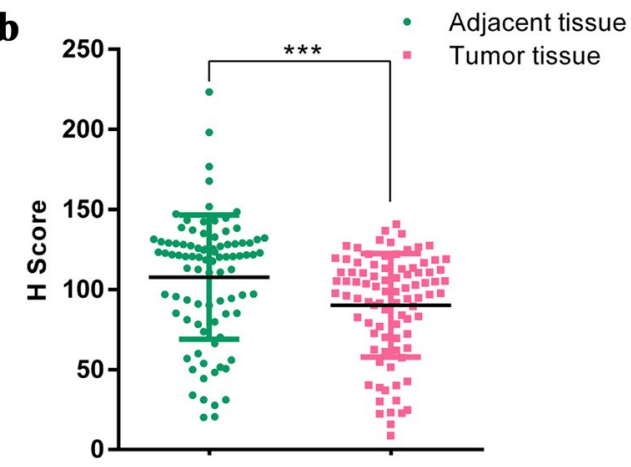

d

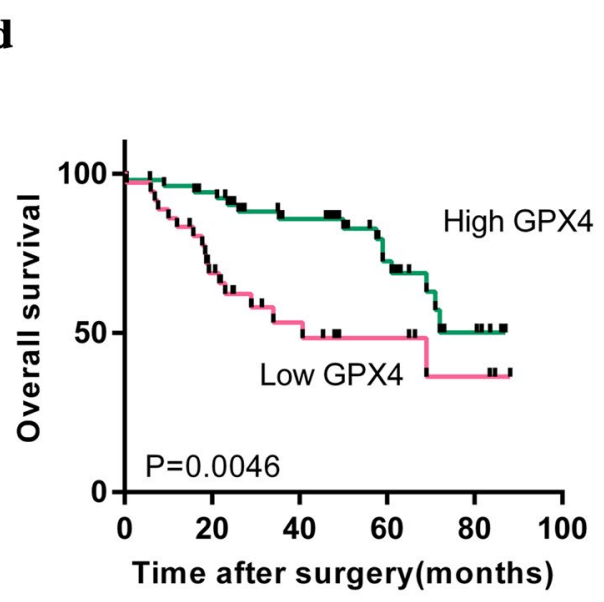

d

\begin{tabular}{|c|c|c|c|c|c|}
\hline \multirow[t]{2}{*}{ Variable } & \multirow[t]{2}{*}{ All case } & \multicolumn{2}{|l|}{ Univariate analysis } & \multicolumn{2}{|l|}{ Multivariate analysis } \\
\hline & & HR $(95 \%$ CI $)$ & $P$ value & HR $(95 \%$ CI) & $P$ value \\
\hline Gender & & $1.043(0.690-2.852)$ & 0.346 & & \\
\hline Male & 57 & & & & \\
\hline Female & 32 & & & & \\
\hline Age (years) & & $1.952(0.970-3.927)$ & 0.061 & & \\
\hline$<60$ & 51 & & & & \\
\hline$\geq 60$ & 38 & & & & \\
\hline Differentiation & & $2.688(1.123-6.431)$ & 0.026 & & \\
\hline Well & 7 & & & & \\
\hline Moderate & 21 & & & & \\
\hline Poor & 61 & & & & \\
\hline PTNM stage & & $2.349(1.333-4.139)$ & 0.003 & $2.035(1.156-3.584)$ & 0.014 \\
\hline $\mathrm{I} / \mathrm{II}$ & 31 & & & & \\
\hline III/IV & 58 & & & & \\
\hline GPX4 expression & & $0.376(0.186-0.761)$ & 0.007 & $0.473(0.228-0.979)$ & 0.045 \\
\hline Low & 36 & & & & \\
\hline High & 53 & & & & \\
\hline
\end{tabular}

HR hazard ratio, 95\% CI 95\% confidence interval; 
Table 2 Correlations between GPX4 protein levels and clinical-pathological parameters of GC patients

\begin{tabular}{lllll}
\hline Variable & All case & GPX4 & \multirow{2}{*}{$P$ value } \\
\cline { 3 - 3 } & & Low no. (\%) & High no. (\%) & \\
\hline Gender & 57 & $20(22.5)$ & $37(41.5)$ & 0.173 \\
Male & 32 & $16(18.0)$ & $16(18.0)$ & \\
Female & & & & \\
Age (years) & & $18(20.2)$ & $33(37.1)$ & 0.256 \\
$<60$ & 51 & $18(20.2)$ & $20(22.5)$ & \\
$\geq 60$ & 38 & & & \\
Differentiation & & $3(3.4)$ & $4(4.5)$ & 0.026 \\
Well & 7 & $3(3.4)$ & $18(20.2)$ & \\
Moderate & 21 & $30(33.7)$ & $31(34.8)$ & \\
Poor & 61 & & & \\
PTNM stage & & $9(10.1)$ & $22(24.7)$ & 0.044 \\
I/II & 31 & $27(30.4)$ & $31(34.8)$ & \\
III/IV & 58 & & & \\
\hline
\end{tabular}

\section{Apatinib further down-regulated GPX4 in GC cell lines}

GPX4 expression was clearly lower in GC tissues than in paracancerous tissues. Gastric cancer (GC) cell lines were more susceptible to ferroptosis than normal cells. Therefore, we hypothesized that apatinib might induce ferroptosis in GC cells via regulation of GPX4. Incubation of MGC-803 and AGS cells with apatinib for $48 \mathrm{~h}$ resulted in down regulation of GPX4 mRNA and protein (Fig. 4a, b). We used MGC803 cells transfected with GPX4 siRNA to develop an MGC803-GPX4-KD cell line. Transfection efficiency was determined using qPCR and western blot (Fig. 4c, d). The cell viability of MGC803-GPX4-KD cells and AGS-GPX4-KD cells was decreased following exposure to apatinib, and this effect was blocked by pretreatment with GSH $(1 \mathrm{mM})$ or vitamin E $(100 \mu \mathrm{M})$ ( Fig. 4e, f). Intriguingly, there seems to be no differences between control and siGPX4 co-incubation. The results suggested that siGPX4 and apatinib have certain overlapping effects on inducing cell death, it is likely to contribute to similar regulation mechanism of siGPX4 and apatinib on the GPX4 transcription. This also suggested that apatinib induced ferroptosis in gastric cancer cells by regulating the transcription of GPX4. We further explore whether apatinib and GPX4 protein function inhibitor RSL3 have complementary effects, it was observed that the apatinib and RSL3 co-incubation has more significant effects on aggravating the cell ferroptosis than the apatinib group (Fig. 4g). These results in vitro agreed with the results of our in vivo experiments.
SREBP-1a regulates GPX4 transcription in gastric cancer cells by binding to the GPX4 promoter region

In a further study, we aimed to determine the regulator that down-regulated GPX4 expression. After a database prediction and gene co-expression analysis, we found a potential candidate regulator, namely SREBP-1a, which is a classical transcription factor that binds to SRE motifs in target gene promoters. We wondered whether SREBP-1a regulates GPX4 expression via its activity as a transcription factor. As shown in Fig. 5a, the expression of SREBP-1a mRNA was decreased in AGS cells following addition of apatinib. Apatinib downregulated the protein levels of activated SREBP-1 (as assessed by the decreased $\mathrm{N}$-terminal cleavage fragment of SREBP-1) (Fig. 5b) [11]. Furthermore, we examined the effects of transfection of active SREBP-1aDelta (a constitutively active form of human SREBP-1) plasmids on GPX4 expression in AGS cells [12]. The data show that overexpression of SREBP-1aDelta upregulated the expression of GPX4 (Fig. 5c, d). In contrast, Inhibition of SREBP1 by betulin or fatostatin decreased the mRNA and protein levels of GPX4 (Fig. 5e, f). These results indicate that SREBP-1a may regulate GPX4 expression at the transcriptional level and down-regulated GPX4 expression after exposed to apatinib. We next examined the GPX4 promoter to better understand how GPX4 is induced by SREBP-1a. We cloned the different fragments of the GPX4 promoters into the pEZX-FR01 expression vector. Expression of SREBP-1aDelta markedly enhanced the luciferase activity driven by the full-length promoter of GPX4. Targeted deletions of this upstream region revealed that the 1,300 and 1,700 bp sub-region was critical for SREBP-1Delta-enhanced promoter activity (Fig. 5g). Chromatin immunoprecipitation (ChIP) show that SREBP-1a bound to this region of the GPX4 promoter (Fig. 5h). Then we analyzed this critical sub-region using the eukaryotic promoter database online promoter analysis tool identified one motif with similarity to transcription factor SRE family binding sites. Deletion of this motif blocked GPX4 promoter activity (Fig. 5g). These results suggest that SREBP-1a directly activates GPX4 gene transcription by binding to a specific locus on the GPX4 promoter.

\section{Apatinib inhibited growth of gastric cancer tumors via down-regulation of GPX4 in vivo}

We evaluated whether apatinib could prevent tumor growth in athymic nude mice implanted with subcutaneous xenograft tumors derived from luciferase-expressing MGC803 cells via down-regulation of GPX4. We hypothesized that apatanib may prevent tumor relapse via inhibition of GPX4. We observed significant reductions in tumor volumes and tumor weights in the groups treated with apatinib and RSL3 compared with those in the groups treated with 
Fig. 4 Apatinib further downregulated GPX4 in GC cell lines. a, b The expression of GPX4 in GC cell lines treated with apatinib or not was evaluated using RT-PCR and western blot. c, d Glutathione peroxidase 4 protein expression was determined using RT-PCR and western blot following transfection of MGC-803 cells with GPX4-siRNA. e, f Cell viability of MGC-803 and AGS cells transfected with GPX4 siRNA and treated with $20 \mu \mathrm{M}$ apatinib with or without GSH (1 mM) or vitamin $\mathrm{E}(100 \mu \mathrm{M})$. $\mathbf{g}$ Cell viability of AGS cells treated with $20 \mu \mathrm{M}$ apatinib and RSL3 $(10 \mu \mathrm{M})$ with or without GSH $(1 \mathrm{mM})$ or vitamin $\mathrm{E}(100 \mu \mathrm{M})$. The error bars represent the standard error from three replicates. $* P<0.05, * * P<0.01$, $* * * P<0.001$ $\mathbf{a}$

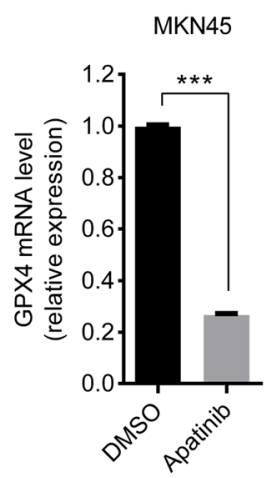

c

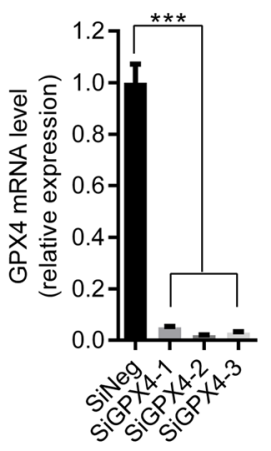

b

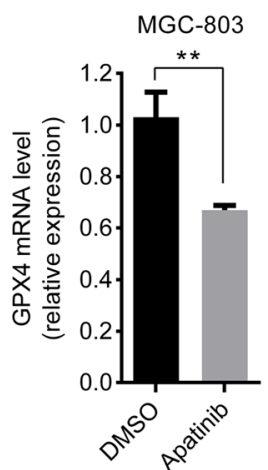

d
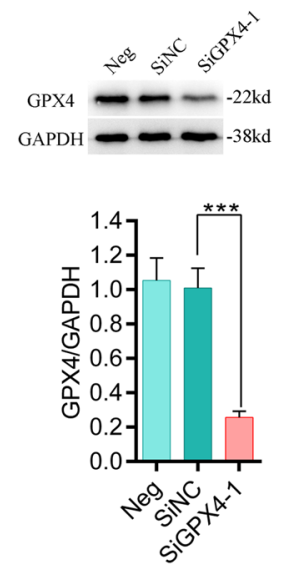

AGS

f

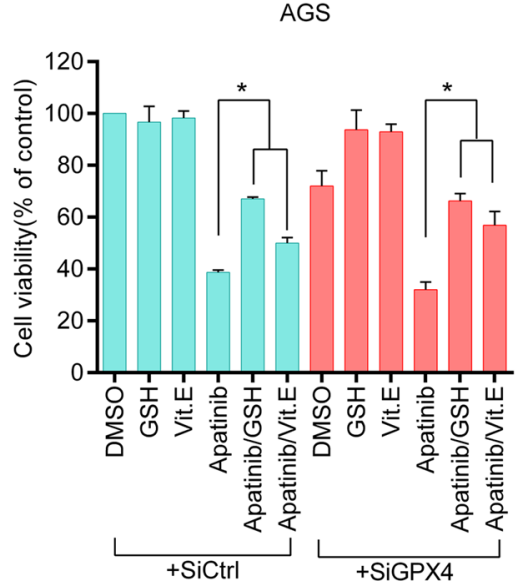

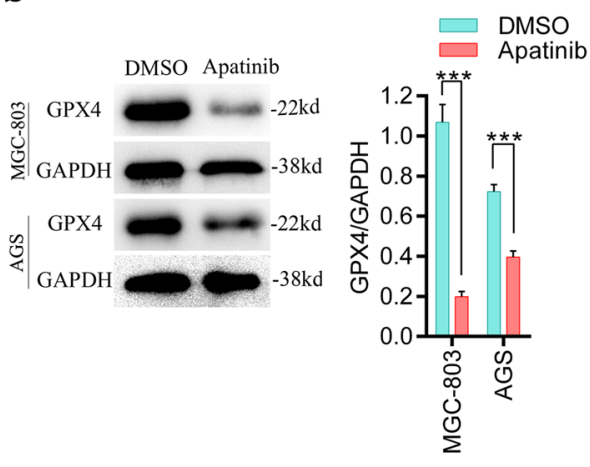

e

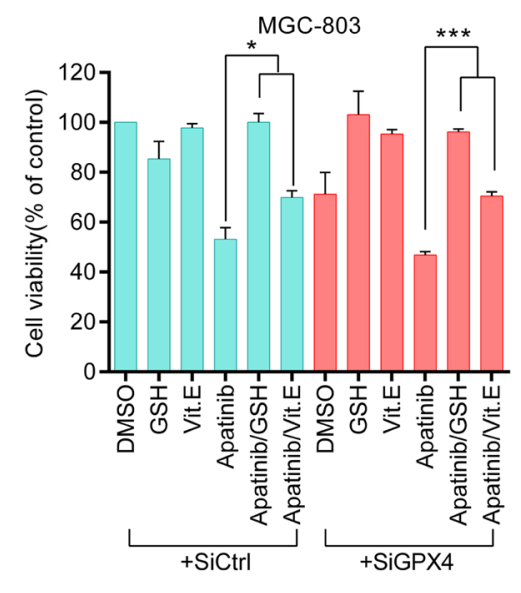

$\mathbf{g}$

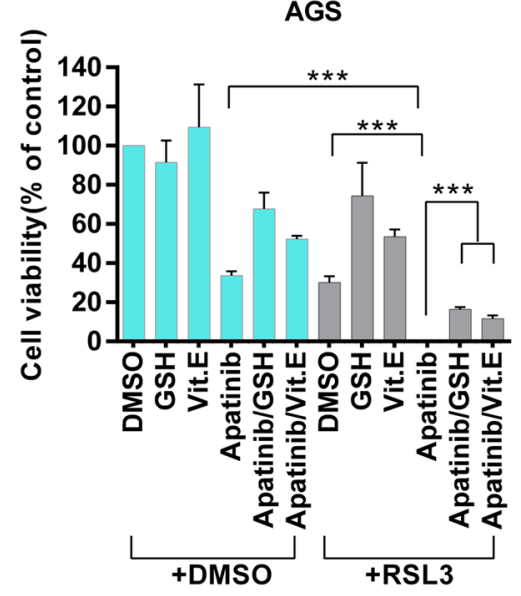

apatinib or RSL3 alone, or in the vehicle-treated control group $(p<0.001)$. Apatinib and vitamin $\mathrm{E}$ co-treatment reduced the inhibitory effect of apatinib on tumor growth $(p<0.001)$ (Fig. 5a, b). To determine whether the antitumor effect of apatinib was associated with ferroptosis, ferric iron was measured in xenograft tumors using Perls staining. The results showed higher concentrations of ferric iron in the apatinib group than those in the vehicle-treated control group (Fig. 5c). Moreover, IHC showed that apatinib treatment resulted in decreased GPX4 expression in tumors (Fig. 5d). These results showed that apatinib treatment decreased the levels of GPX4 in vivo, which agreed with the results of our in vitro experiments.

\section{Multi-drug-resistant (MDR) GC cells were vulnerable to apatinib-induced inhibition of GPX4}

To determine whether cancer cells in a high-mesenchymal therapy-resistant state were more sensitive to apatinibinduced ferroptosis, cisplatin-resistant and 5-FU-resistant 
Fig. 5 SREBP-1a regulates GPX4 transcription in gastric cancer cells by binding to the GPX4 promoter region. a, b Real-time PCR and western blot analysis of AGS cells after treated with apatinib expressing the N-terminal SREBP-1a (nSREBP-1a) for $48 \mathrm{~h} . \mathbf{c}, \mathbf{d}$ Real-time PCR and western blot analysis of AGS cells after infection with the active SREBP-1aDelta plasmids for $48 \mathrm{~h}$. e, f Glutathione peroxidase 4 expression was determined in AGS cells treated with betulin $(8 \mu \mathrm{M})$, fatostatin $(1.5 \mu \mathrm{M})$ or DMSO for $48 \mathrm{~h}$, using RT-PCR and western blot. g Luciferase activity analysis of different fragments of the GPX4 promoters cloned in the pEZX-FR01-basic vector that were transfected into AGS cells together with PC3.1 and SREBP-1aDelta plasmids for 48 h. f Chromatin immunoprecipitation using specific antibodies showed the binding of SREBP-1a to the GPX4 promoter $(-1,700$ to $-1,300 \mathrm{bp})$.

The error bars represent the standard error from three replicates. $* P<0.05, * * P<0.01$, $* * * P<0.001$ a

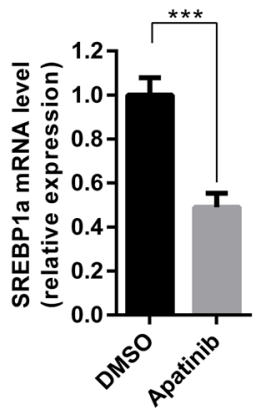

c
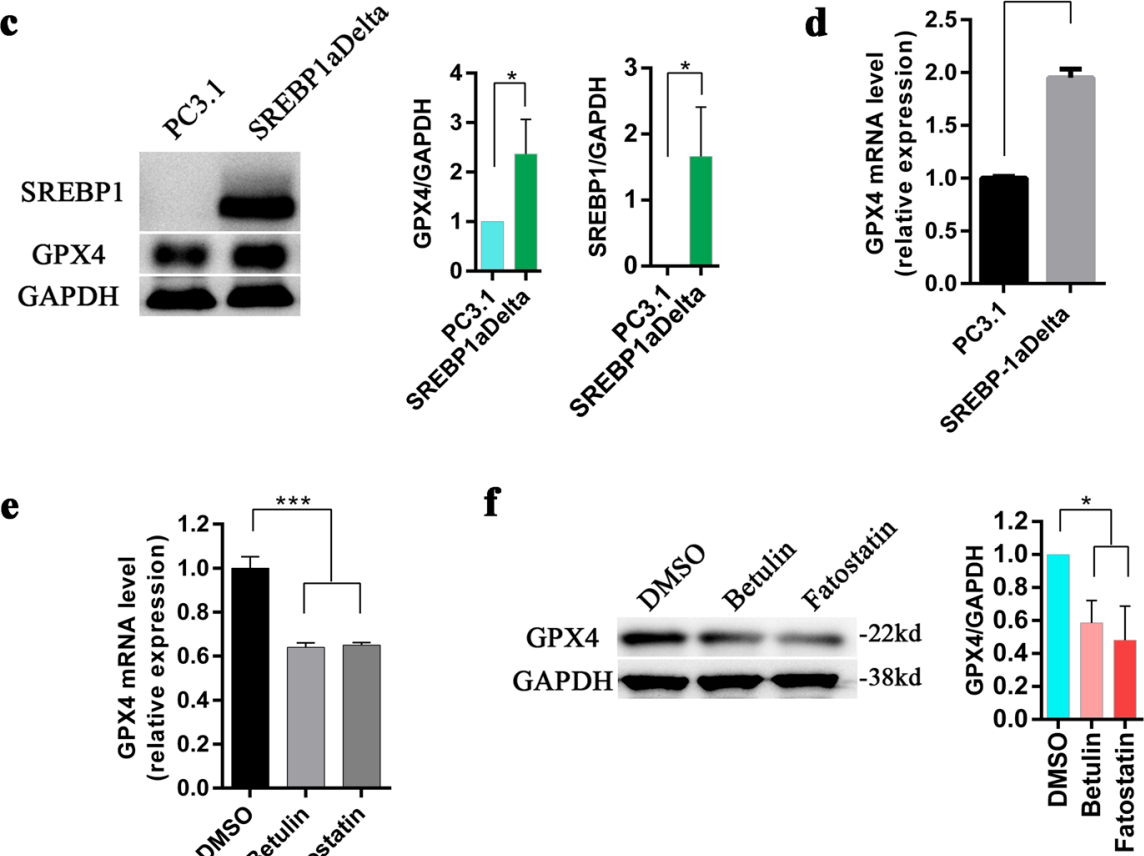
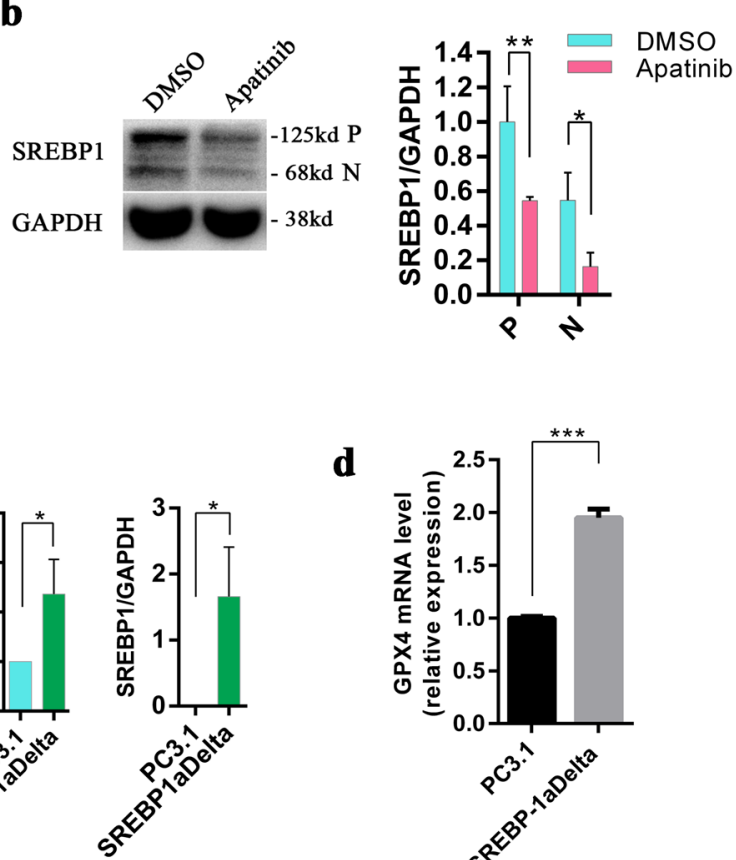

d

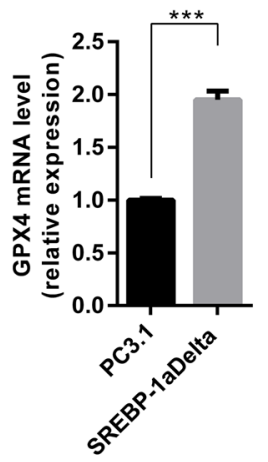

e

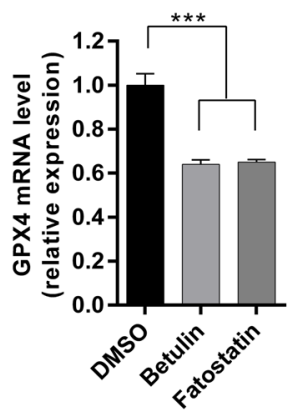

f

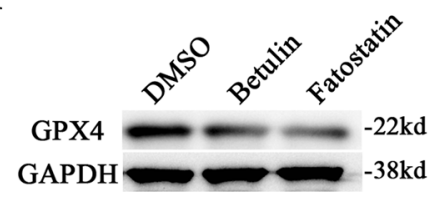

g

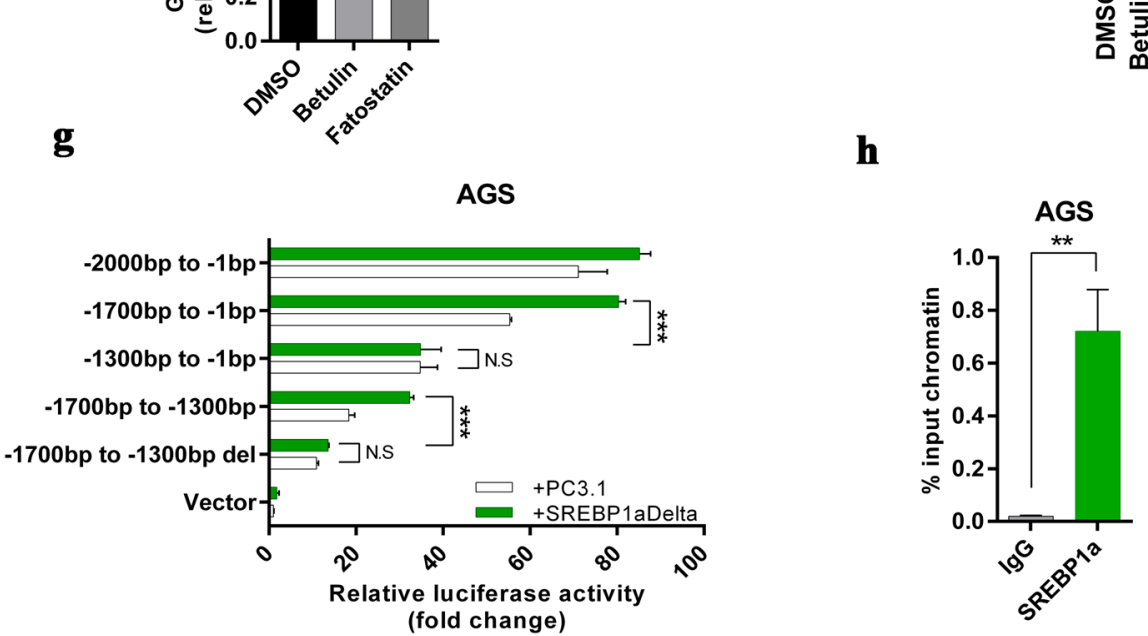

MGC-803/DDP/5-FU cells were generated from the parental MGC-803 cells. The viability of (1S, 3R)-RSL3-treated MGC-803/DDP/ 5-FU cells decreased in a time-dependent manner, and this effect was markedly blocked by co-incubation with ferrostatin-1 $(10 \mu \mathrm{M})(p<0.01)$ (Fig. 6a). Furthermore, MGC-803/DDP/5-FU cells were more sensitive to apatinib than the parental MGC-803 cells (Fig. 6b). Iron assay kit results showed significantly higher concentrations of total and ferrous iron in MGC-803/DDP/5-FU cells treated with $20 \mu \mathrm{M}$ apatinib compared with the parental MGC-803 cells $(p<0.05)$ (Fig. 6c). Co-treatment of apatinib /iron showed more pronounced inhibition in cell survival, as compared to apatinib treatment alone $(p<0.01)$ (Fig. 6d). In addition, we found that GPX4 was down-regulated in the apatinib-treated group compared with that in the DMSO control group (Fig. 6e). In MGC-803/DDP/ 5-FU cells treated with apatinib, cellular MDA levels and cellular ROS levels were significantly increased compared with those 
Fig. 6 Apatinib inhibited growth of GC tumors via down-regulation of GPX4 in vivo. a MGC-803 cells were implanted subcutaneously in nude mice $(n=6)$. Representative images of mice and luciferase signal intensities are shown. b Tumor volumes were recorded at the indicated times. c Ferric iron was detected using Perls staining $(400 \times)$. d Representative IHC images of GPX4 in the tumors $(200 \times$ and $400 \times)$. $* P<0.05, * * P<0.01$, $* * * P<0.001$ $\mathbf{a}$

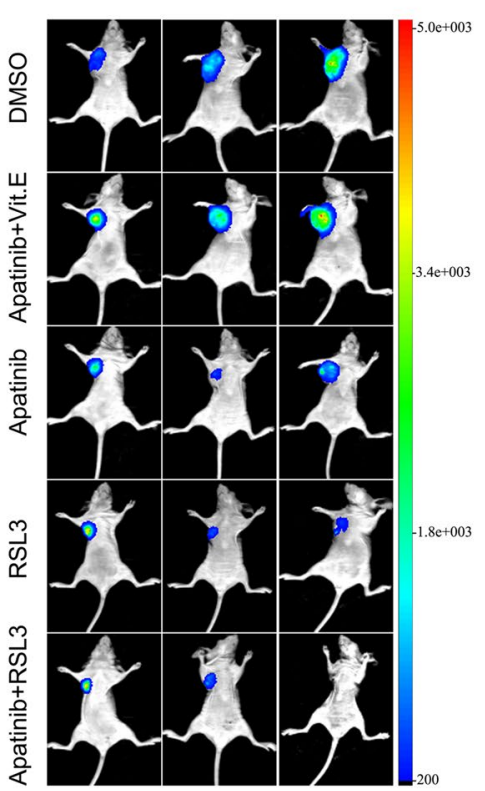

c
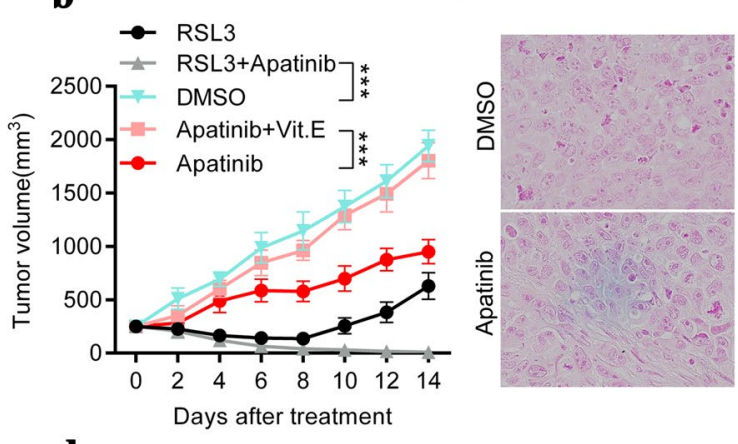

d
Apatinib

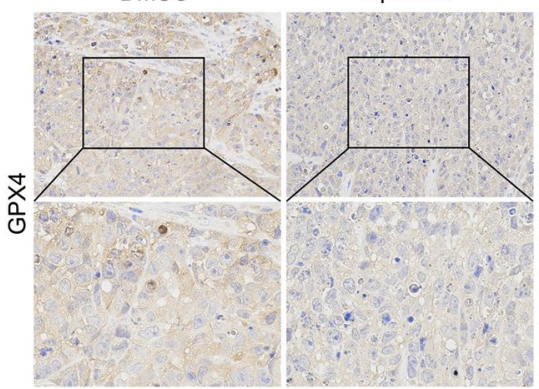

in the DMSO control group $(p<0.01)$. These effects were blocked by pretreatment with ferrostatin-1 (Fig. 6f). Cellular GSH levels were strongly decreased in MGC-803/DDP/5FU cells treated with apatinib and transfected with siGPX4 compared with those in siRNA control cells or cells treated with apatinib alone $(p<0.001)$ (Figs. 6g, 7).

\section{Discussion}

Apatinib has shown promising antitumor effects against metastatic GC and a number of other tumors [2, 14, 15]. However, it still cannot predict the drug efficacy of apatinib, therefore, the mechanism of apatinib anticancer treatment need to be clarified. Oxidative stress occupies a critical role in the regulation of various biological processes including cell death and innate immunity. Here, we demonstrated a potential involvement of GPX4 in coordinating lipid peroxidation and ferroptosis in apatinib anticancer treatment. In response to apatinib treatment, GPX4 is downregulated in GC cells to accelerate apatinib-mediated ferroptotic cell death, thereby enhancing the efficacy of apatinib (Fig. 6h). These findings establish GPX4 as an essential negative regulator of ferroptosis in apatinib anticancer treatment.

Glutathione peroxidase 4 is considered the most important member of the GPX family because it catalyzes the reduction of lipid peroxides, and knockout of GPX4 is lethal to the embryos of mouse model [16-18]. GPX4 has emerged as the main endogenous inhibitor of ferroptosis, and acts via GSH-dependent inhibition of lipid peroxidation-induced cell membrane damage [10, 19]. Using IHC, we showed that GPX4 was significantly down-regulated in GC tissues compared with that in adjacent normal tissues (Fig. 3a, b). Low-level expression of GPX4 may be an independent risk factor for poor prognosis and OS in patients with GC. Reduced expression of GPX4 may result in decreased ability to eliminate ROS, and increased ROS production results in increased stimulation of proliferation, invasion, migration, and angiogenesis [20]. In addition, slightly elevated ROS levels may promote the development of mechanisms by cancer cells to evade apoptosis. This may result in cancer cells that are refractory to a broad range of anti-cancer therapies. Beside, Glutathione peroxidase 4-mediated regulation of cyclooxygenase and lipoxygenase activities may contribute to early stages of inflammation-mediated carcinogenesis [21]. Although this metabolic change supports growth and proliferation, it exposes the Achilles' heel of cancer cells, excessive ROS causes lipid peroxidation and increases susceptibility to ferroptosis [22]. GPX4 deletion was shown to result in extensive lipid peroxidation, leading to lethality or cell loss. In a further study, we found a potential candidate regulator, namely SREBP-1a, which is a classical transcription factor that binds to SRE motifs in target gene promoters. We proved that SREBP-1a could regulate GPX4 expression and validated an effective binding site (located at $-1655 \sim-1645$ bp relative to the TSS).

Glutathione peroxidase 4 is a promising target gene for GC therapy. Apatinib has been successfully repurposed as a second-line treatment option for patients with advanced or metastatic GC, and is considered to be generally safe and 
Fig. 7 Multi-drug-resistant GC cells were vulnerable to apatinib-induced GPX4 inhibition. a Cell viability was assessed following exposure of MGC-803/DDP/5-FU cells to $10 \mu \mathrm{M}$ RSL3, $10 \mu \mathrm{M}$ Fer-1, or both. b Cell viability was assessed following treatment of MGC-803/DDP/5-FU and MG-803 cells with apatinib. c MGC-803/DDP/5-FU and MG-803 cells were incubated with $20 \mu \mathrm{M}$ apatinib for $24 \mathrm{~h}$, and total and ferrous iron was detected using an iron assay kit. $d$ The addition of $100 \mu \mathrm{M}$ FeSO 4 to $10 \mu \mathrm{M}$ apatinib-induced cell death. e Glutathione peroxidase 4 expression was determined in MGC-803/DDP/5-FU cells treated with apatinib or DMSO for $48 \mathrm{~h}$, using western blot. $\mathrm{f}$ Changes in cellular MDA levels were evaluated in MGC-803/ $\mathrm{DDP} / 5-\mathrm{FU}$ cells treated with $20 \mu \mathrm{M}$ apatinib, $10 \mu \mathrm{M}$ Fer-1, or both. g Cellular GSH levels were measured in control cells as well as in GPX4 siRNAtransfected MGC-803/DDP/5FU cells following treatment with apatinib. h Schematic diagram summarizing the role of apatinib in lipid peroxidation and ferroptosis. $* P<0.05$, $* * P<0.01, * * * P<0.001$ $\mathbf{a}$

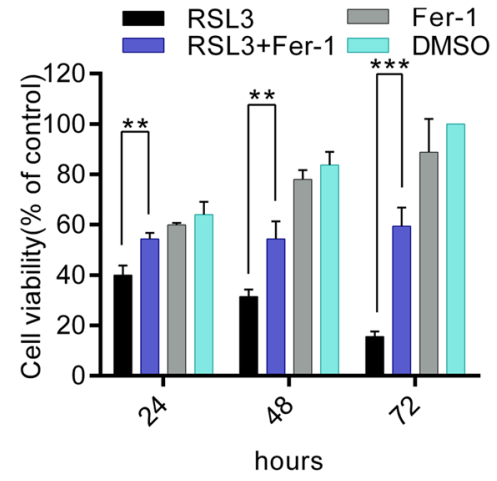

c

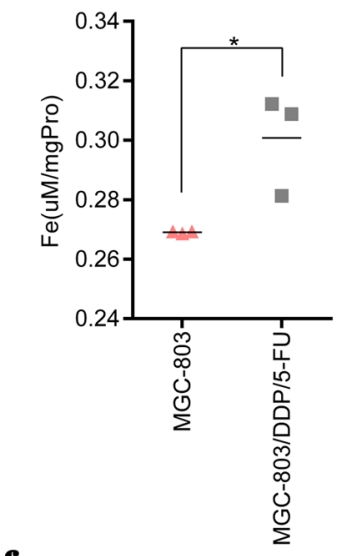

$\mathbf{f}$

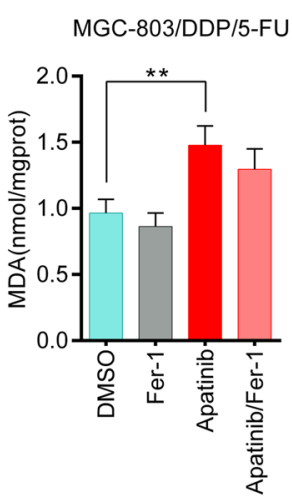

d

MGC-803/DDP/5-FU

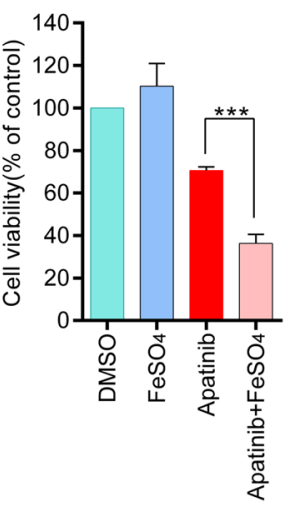

g
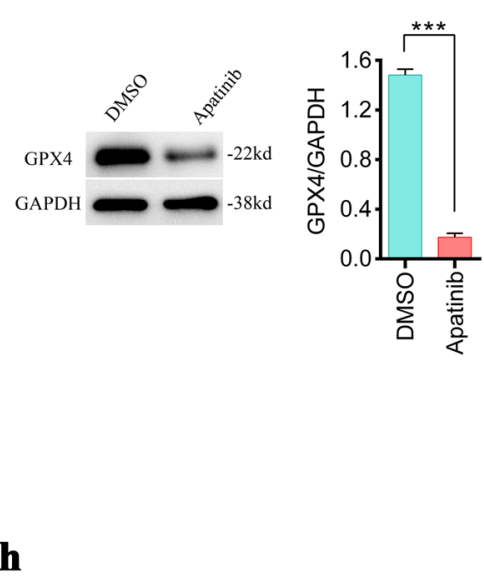

e b

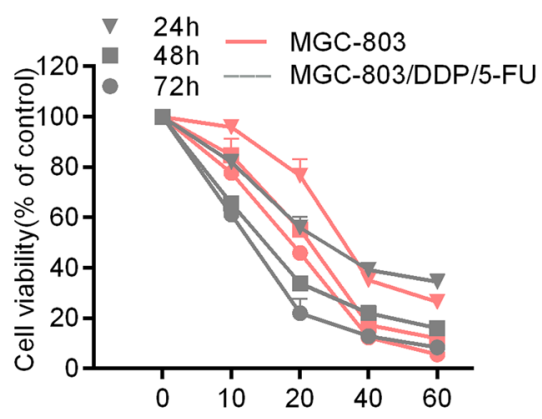

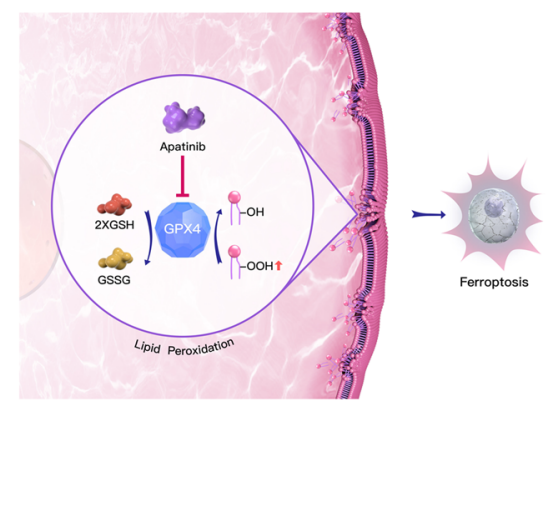

well tolerated $[2,23]$. As well known, four mechanisms of initiation of ferroptosis have been identified. Erastin, sulfasalazine, and sorafenib are small-molecule class 1 ferroptosis inducers that starve cells of the amino acid cysteine [24]. Class 2 ferroptosis inducers act by direct inhibition of GPX4. A common class 2 ferroptosis inducer is the small molecule (1S, 3R)-RSL3 (RSL3), which directly inhibits GPX4, leading to cell death $[10,25,26]$. Class 3 ferroptosis inducers, such as FIN56 and CIL56, act by depleting GPX4 protein and mevalonate-derived coenzyme Q10 (CoQ10) $[27,28]$. The class 4 ferroptosis inducer FINO2, the only member of this class, acts by oxidizing iron [29]. However, many ferroptosis inducers were not suitable for in vivo use owing to their low solubility or the difficulties associated with characterization of its pharmacokinetic parameters [30]. Our study showed that apatinib down-regulated GPX4 in gastric cancer cells, resulting in ferroptosis, it works in the similar way as class 3 ferroptosis inducers. Our study provides a further characterization of the molecular mechanisms by which apatinib exerts its therapeutic effects.

A previous study showed that drug-resistant cancer cells that have undergone epithelial-to-mesenchymal transition are particularly sensitive to GPX4 inhibitors [31]. Effective GPX4 inhibitors could be reduces the residual persister 
cells after conventional chemotherapy [32]. Our results were consistent with this finding. We found that multi-drug-resistant (MDR) GC cells were vulnerable to apatinib-induced GPX4 inhibition (Fig. 6b-d). Drug resistance is often associated with resistance to drug-induced apoptosis [33, 34]. Nonapoptotic forms of cell death may facilitate selective elimination of some tumor cells, and induction of these forms of cell death may be a promising strategy to eliminate cancer cells resistant to drug-induced apoptosis [35]. Our results suggest that drug-resistant cancer cells were vulnerable to undergo ferroptosis, a non-apoptotic form of cell death. More broadly, the results presented here illustrate a new molecular mechanism to understand how apatinib sensitizes resistant tumor cells to chemotherapy drugs and can reverse the multiple drug resistance (MDR).

\section{Conclusion}

In summary, our study showed that GPX4 was down-regulated in GC tissues, which was associated with poor prognosis. Apatinib exerted antitumor effects against GC cells in vitro and in vivo through the induction of lipid peroxidation mediated by GPX4, then lead to ferroptosis. Furethermore, we found apatinib inhibited transcription of GPX4 via a SREBP1a-mediated pathway. These results indicated that GPX4 may be a potential target for anti-GC efficacy evaluation and treatment of apatinib.

Supplementary Information The online version contains supplementary material available at https://doi.org/10.1007/s10120-021-01159-8.

Author contributions QM, GXL, and LYZ conceived of and designed the experiments. LYZ, YMP, SXH, RL, ZQW, JHH, and XTL performed the experiments. LYZ, YMP and SXH analyzed the data. QM contributed reagents/materials/analysis tools. LYZ and YMP wrote the draft, and made all revisions. All authors read and approved the final manuscript.

Funding This work was supported by the National Natural Science Foundation of China (81772133 to QM and 81902444 to LYZ); the Guangdong Natural Science Fund (2020A1515010269 to LYZ and 2020A1515011367 to QM); the Guangzhou Citizen Health Science and Technology Research Project (201803010034 to QM and 201903010072 to LYZ).

Availability of data and materials All data and details can be obtained by contacting the corresponding author.

\section{Compliance with ethical standards}

Conflict of interest The authors declare that they have no conflict of interest.

Ethics approval and consent to participate All procedures involving animals and their care in this study were undertaken in accordance with the National Institutes of Health Guide for the Care and Use of
Laboratory Animals, and approved by the Southern Medical University Institutional Animal Care and Use Committee.

Consent for publication All authors agree the publication of this study.

\section{References}

1. Brower V. Apatinib in treatment of refractory gastric cancer. Lancet Oncol. 2016;17:e137.

2. Li J QSXJ. Randomized, Double-Blind, Placebo-Controlled Phase III Trial of Apatinib in Patients With Chemotherapy-Refractory Advanced or Metastatic Adenocarcinoma of the Stomach or Gastroesophageal Junction.; 2016.

3. Fathi MN, Rashidi MR, Vahedian V, Akbarzadeh M, Fattahi A, Nouri M. Therapeutic potentials of Apatinib in cancer treatment: Possible mechanisms and clinical relevance. Life Sci. 2020;241:117106.

4. Zhang H. Apatinib for molecular targeted therapy in tumor. Drug Des Devel Ther. 2015;9:6075-81.

5. Zhao D, Hou H, Zhang X. Progress in the treatment of solid tumors with apatinib: a systematic review. Oncol Targets Ther. 2018;11:4137-47.

6. Gaschler MM, Stockwell BR. Lipid peroxidation in cell death. Biochem Biophys Res Commun. 2017;482:419-25.

7. Dixon SJ, Lemberg KM, Lamprecht MR, Skouta R, Zaitsev EM, Gleason CE, et al. Ferroptosis: an iron-dependent form of nonapoptotic cell death. Cell. 2012;149:1060-72.

8. Shen Z, Song J, Yung BC, Zhou Z, Wu A, Chen X. Emerging strategies of cancer therapy based on ferroptosis. Adv Mater. 2018;30:e1704007.

9. Yu H, Guo P, Xie X, Wang Y, Chen G. Ferroptosis, a new form of cell death, and its relationships with tumourous diseases. J Cell Mol Med. 2017;21:648-57.

10. Yang WS, SriRamaratnam R, Welsch ME, Shimada K, Skouta R, Viswanathan VS, et al. Regulation of ferroptotic cancer cell death by GPX4. Cell. 2014;156:317-31.

11. Cheng C, Ru P, Geng F, Liu J, Yoo JY, Wu X, et al. Glucose-mediated N-glycosylation of SCAP is essential for SREBP-1 activation and tumor growth. Cancer Cell. 2015;28:569-81.

12. Harada N, Yonemoto H, Yoshida M, Yamamoto H, Yin Y, Miyamoto A, et al. Alternative splicing produces a constitutively active form of human SREBP-1. Biochem Biophys Res Commun. 2008;368:820-6.

13. Li J, Qin S, Xu J, Guo W, Xiong J, Bai Y, et al. Apatinib for chemotherapy-refractory advanced metastatic gastric cancer: results from a randomized, placebo-controlled, parallel-arm, phase II trial. J Clin Oncol. 2013;31:3219-25.

14. Tian S, Quan H, Xie C, Guo H, Lü F, Xu Y, et al. YN968D1 is a novel and selective inhibitor of vascular endothelial growth factor receptor-2 tyrosine kinase with potent activity in vitro and in vivo. Cancer Sci. 2011;102:1374-80.

15. Ran Q, Van Remmen H, Gu M, Qi W, Roberts LJN, Prolla T, et al. Embryonic fibroblasts from Gpx4+/- mice: a novel model for studying the role of membrane peroxidation in biological processes. Free Radical Biol Med. 2003;35:1101-9.

16. Esworthy RS, Aranda R, Martín MG, Doroshow JH, Binder SW, Chu FF. Mice with combined disruption of Gpx 1 and Gpx2 genes have colitis. American journal of physiology. Gastrointestinal and liver physiology 2001;281: G848-55.

17. Imai $\mathrm{H}$, Nakagawa $\mathrm{Y}$. Biological significance of phospholipid hydroperoxide glutathione peroxidase (PHGPx, GPx4) in mammalian cells. Free Radical Biol Med. 2003;34:145-69. 
18. Agmon E, Solon J, Bassereau P, Stockwell BR. Modeling the effects of lipid peroxidation during ferroptosis on membrane properties. Sci Rep UK. 2018;8:5155.

19. Panieri E, Santoro MM. ROS homeostasis and metabolism: a dangerous liason in cancer cells. Cell Death Dis. 2016;7:e2253.

20. Brigelius-Flohe R, Kipp A. Glutathione peroxidases in different stages of carcinogenesis. Biochim Biophys Acta. 2009;1790:1555-68.

21. Gorrini C, Harris IS, Mak TW. Modulation of oxidative stress as an anticancer strategy. Nat Rev Drug Discov. 2013;12:931-47.

22. Hicklin DJ, Ellis LM. Role of the vascular endothelial growth factor pathway in tumor growth and angiogenesis. J Clin Oncol. 2005;23:1011-27.

23. Coriat R, Nicco C, Chéreau C, Mir O, Alexandre J, Ropert S, et al. Sorafenib-induced hepatocellular carcinoma cell death depends on reactive oxygen species production in vitro and in vivo. Mol Cancer Ther. 2012;11:2284-93.

24. Yang WS, Kim KJ, Gaschler MM, Patel M, Shchepinov MS, Stockwell BR. Peroxidation of polyunsaturated fatty acids by lipoxygenases drives ferroptosis. Proc Natl Acad Sci USA. 2016;113:E4966-75.

25. Yang WS, Stockwell BR. Synthetic lethal screening identifies compounds activating iron-dependent, nonapoptotic cell death in oncogenic-RAS-harboring cancer cells. Chem Biol. 2008;15:234-45.

26. Shimada K, Skouta R, Kaplan A, Yang WS, Hayano M, Dixon SJ, et al. Global survey of cell death mechanisms reveals metabolic regulation of ferroptosis. Nat Chem Biol. 2016;12:497-503.
27. Bentinger M, Brismar K, Dallner G. The antioxidant role of coenzyme Q. Mitochondrion. 2007;7(Suppl):S41-50.

28. Gaschler MM, Andia AA, Liu H, Csuka JM, Hurlocker B, Vaiana CA, et al. FINO(2) initiates ferroptosis through GPX4 inactivation and iron oxidation. Nat Chem Biol. 2018;14:507-15.

29. Feng H, Stockwell BR. Unsolved mysteries: How does lipid peroxidation cause ferroptosis? PLoS Biol. 2018;16:e2006203.

30. Viswanathan VS, Ryan MJ, Dhruv HD, Gill S, Eichhoff OM, Seashore-Ludlow B, et al. Dependency of a therapy-resistant state of cancer cells on a lipid peroxidase pathway. Nature. 2017;547:453-7.

31. Hangauer MJ, Viswanathan VS, Ryan MJ, Bole D, Eaton JK, Matov A, et al. Drug-tolerant persister cancer cells are vulnerable to GPX4 inhibition. Nature. 2017;551:247-50.

32. Igney FH, Krammer PH. Death and anti-death: tumour resistance to apoptosis. Nat Rev Cancer. 2002;2:277-88.

33. Song J. EMT or apoptosis: a decision for TGF-beta. Cell Res. 2007;17:289-90.

34. Speirs CK, Hwang M, Kim S, Li W, Chang S, Varki V, et al. Harnessing the cell death pathway for targeted cancer treatment. Am J Cancer Res. 2011;1:43-61.

Publisher's Note Springer Nature remains neutral with regard to jurisdictional claims in published maps and institutional affiliations. 